\title{
„Alles nur Schall, alles nur Rauch“
}

\author{
Wie Popmusik über (ihre) Vergänglichkeit nachdenkt
}

\begin{abstract}
Beim Aufrufen des Vanitas-Motivs in der Popmusik wird nicht nur die Vergänglichkeit des menschlichen Daseins thematisiert, sondern auch die Kurzlebigkeit populärer Musik selbst: Popsongs bedauern ihre Abhängigkeit von Aufmerksamkeitskonjunkturen, erinnern an den unausweichlichen ,Schlussakkord' und arbeiten dennoch an ihrer Archivierung. Auch jenseits der Lyrics tritt das Vanitas-Motiv in Erscheinung - auf Plattencovern, in Szene-Ikonographien auf Kleidungsstücken oder unter der Haut. Ausgehend von diesen Beobachtungen und anknüpfend an Diagnosen zur Pop-Retromanie geht der Beitrag dem selbstreferentiellen Potential des ,Endes' im Pop nach, indem er unterschiedliche Aufrufungen und Semantisierungen, abwandelnde Wiederholungen und dekorhafte Stilisierungen von Vanitas in verschiedenen Formen und Formationen populärer Musik in den Blick nimmt.
\end{abstract}

\section{Todesbesessenheit: Vergänglichkeit im Pop, Vergänglichkeit des Pop ${ }^{1}$}

Im Januar 2018 erschien im Online-Businessmagazin Quartz eine Kolumne zur damals kurz bevorstehenden Grammy-Verleihung mit dem Titel: „Pop music has become obsessed with death“. Die beiden Autor^innen, Amy X. Wang und Dan Kopf, schreiben darin: „When the Grammy Awards come on next weekend, nominations for one of the most distinguished honors - song of the year - will spotlight a track not about love or lust or fame, but dying. Dying by one's own hand, to be specific." ${ }^{2}$ Mit dieser Wahl der Nominierung, so Wang und Kopf, trage auch die Grammy-Verleihung einer sich deutlich abzeichnenden Entwicklung in der populären Musik Rechnung, die sich in der Tat immer deutlicher und differenzierter mit Fragen von Tod und Vergänglichkeit auseinandersetze.

1 ,Pop` wird hier als genreübergreifendes Kürzel des Begriffs ,Popmusik‘ (im Weiteren auch ,populäre Musik') verwendet und beschreibt eine sich in diskursiven und nicht-diskursiven Praktiken manifestierende Form musikalischen Ausdrucks, für die deren Einbettung in kommerzielle Produktions-, Verbreitungs- und Konsumptionsmechanismen konstitutiv ist.

2 Amy X. Wang und Dan Kopf. „Pop Music Has Become Obsessed with Death“. Quartz (26.01.2018). https://qz.com/quartzy/1078377/pop-music-has-a-new-obsession-with-death-and-decay/ (Zugriff am 23.10.2020).

Ә Open Access. ( 2021 Martin Butler, publiziert von De Gruyter. (c) BY-NC-ND Dieses Werk ist lizensiert unter einer Creative Commons Namensnennung-Nicht-kommerziell-Keine Bearbeitung 4.0 International Lizenz. https://doi.org/10.1515/9783110716016-009 
So trägt der als ,bestes Lied des Jahres“ nominierte Song, den die beiden ansprechen, den Titel „1-800-273-8255“ - die Nummer der US-amerikanischen telefonischen Seelsorge für Menschen mit suizidalen Tendenzen, der National Suicide Prevention Lifeline. Der Song entstand in einer Zusammenarbeit des US-amerikanischen Rappers Logic, dem ebenfalls aus den Vereinigten Staaten stammenden Singer/Songwriter Khalid und der kanadischen R\&B-Sängerin Alessid und hielt sich wochenlang in den US-Charts.

Die Nominierung gerade dieses Songs nahm das Magazin Quartz zum Anlass für eine Suche nach weiteren Liedern, die sich mit dem Thema Tod und Vergänglichkeit auseinandersetzen. Dazu wurden 15.000 Popsongs untersucht, die seit 1958 in den Billboard Charts unter den ersten hundert Rängen zu finden waren. Das Ergebnis scheint unmissverständlich: Anscheinend gibt es in der Tat eine in der Tendenz ansteigende Zahl von Songs, die sich der Vergänglichkeit des Daseins widmen. Interessant ist dabei nicht so sehr die zu dieser Erhebung verwendete Methode einer qualifizierten Stichwortsuche, über deren Angemessenheit sicher zu diskutieren wäre. Spannend ist vielmehr die Diagnose der Autor^innen. Sie erklären die steigende Faszination für den Tod in der Popmusik seit den 1980er Jahren u. a. mit der Popularisierung von Hip-Hop, der sich gerade in der jüngeren Vergangenheit verstärkt über die Endlichkeit des Lebens Gedanken gemacht habe - mit den Worten der im Artikel zitierten Kulturkritikerin Carrie Battan: „If hip hop has historically focussed on invincibility, this generation is fixated on mortality. Nihilism, taken to an extreme that feels almost competitive, has become its own form of braggadocio“; ${ }^{3}$ die zur Schau gestellte übersteigerte Lust am Vergänglichen ist also mittlerweile schon eine etablierte Spielart der im Rap üblichen Praxis der gegenseiten Selbstüberbietung.

Außerdem, so konstatieren Wang und Kopf, habe sich dieser Impuls zur Thematisierung von Vergänglichkeit und Vergeblichkeit auch in andere Genres des Pop ausgebreitet. Dass diese Themen schon seit den Anfängen kommerzieller Popmusik in den 1950er und 1960er Jahren immer wieder aufgegriffen werden, sei unbenommen, so die Autor^innen. Die in der jüngeren Vergangenheit zu beobachtende eingehende und genreübergreifende Beschäftigung damit reagiere allerdings auf eine noch junge Tendenz zur Enttabuisierung der Themen Schmerz, Tod und Selbstmord im öffentlichen Diskurs - eine Entwicklung, auf die populäre Musik reagiere, und der auch durch populäre Musik Gestalt gegeben werde.

Der vorliegende Beitrag nimmt diese Beobachtungen zur ,Todesbesessenheit‘ des Pop zum Anlass und Ausgangspunkt für eine Erkundung unterschied-

3 Ebd. 
licher Aufrufungen und Semantisierungen, abwandelnden Wiederholungen und dekorhaften Stilisierungen des Motivs der Vergänglichkeit in verschiedenen Formen und Formationen populärer Musik. Er erhebt dabei keinen Anspruch auf Vollständigkeit - zu groß ist die Bandbreite der Variationen, zu facettenreich sind die verschiedenen medialen und materialen Konstellationen, in denen das Motiv auftaucht. ${ }^{4}$ Vielmehr geht der Beitrag stichprobenartig und kursorisch vor und beleuchtet so nur Ausschnitte. Ziel ist daher auch keine umfassende Aufarbeitung der Formen und Funktionen des Vergänglichkeitstopos in populärer Musik, sondern eine durch eine ganz andere Diagnose orientierte und motivierte Zuspitzung der Diagnose nämlich, dass die Beschäftigung mit Vergänglichkeit in populärer Musik vor allem auch als Beschäftigung populärer Musik mit sich selbst zu verstehen ist. Dieser Streifzug geht somit von der Annahme aus, dass die Faszination populärer Musik für das, was sich als mehr oder weniger lose Referenzen auf das barocke Vanitas-Motiv verstehen lässt, nicht nur, aber insbesondere auch als Ausdruck der - schon oft und immer wieder konstatierten - Flüchtigkeit des Populären zu verstehen ist. Nachdenken über Vergänglichkeit hat im Pop also nicht selten ein höchst selbstreflexives Moment. In Zeiten, in denen im öffentlichen und akademischen Diskurs regelmäßig das Ende der Popkultur heraufbeschworen wird man denke nur an Simon Reynolds' Diagnose einer zukunftslosen Retromanie $^{5}$-, scheint dieses Moment des Selbstbezugs besonders stark ausgeprägt zu sein. Damit zumindest ließe sich die von Wang und Kopf beobachtete zunehmende Hinwendung zum Thema Tod und Vergänglichkeit mindestens ebenso gut erklären.

4 Erstaunlichlicherweise ist das Thema in der kulturwissenschaftlichen Forschung bisher nur in Ansätzen systematisch bearbeitet worden, zu den wenigen einschlägigen umfassenderen Erschließungen gehören: Christopher Partridge. Mortality and Music: Popular Music and the Awareness of Death. London u. a.: Bloomsbury, 2015, oder auch die jüngst erschienene Beitragssammlung Dancing with Mr. D: Tod in Popmusik und Kunst. Hrsg. von Jörg Vögele u. a. Köln: Wienand, 2019. Darüber hinaus wird das Thema entweder im Rahmen breiter aufgestellter Einzelbeiträge diskutiert (vgl. etwa Keith Durkin. „Death, Dying, and the Dead in Popular Culture“. Handbook of Death and Dying. Hrsg. von Clifton D. Bryant und Dennis L. Peck. Thousand Oaks: Sage Publications, 2003. 43-49); in Einzelstudien zu bestimmten Genres aufgegriffen (vgl. etwa Brian Lee Cooper. „From Johnny Ace to Frank Zappa: Debating the Meaning of Death in Rock Music.“ Popular Culture in Libraries 3 (1995): 51-75; Deloris von Nordheim. „Visions of Death in Rock Music and Musicians.“ Popular Music and Society 17.2 (1993): 21-31); oder aus anderer (sozialwissenschaftlicher bzw. sozialpsychologischer) Perspektive beleuchtet, vgl. etwa John C. Thrush u. a. „The Concept of Death in Popular Music: A Social Psychological Perspective“. Popular Music and Society 6 (1979): 219-228.

5 Simon Reynolds. Retromania. Warum Pop nicht von seiner Vergangenheit lassen kann. Übers. von Chris Wilpert. Mainz: Ventil, 2012. 
Mit anderen Worten: Auch im Pop ist alles nur Windhauch. Das ,Wir‘ in Kansas’ Klassiker „All We Are is Dust in the Wind“ (zum Beispiel) lässt sich folglich nicht nur lesen als das musikalisch evozierte Wir einer Generation, die sich ihrer eigenen Vergänglichkeit und der Vergeblichkeit ihres Tuns bewusst ist, sondern auch als das musikmachende Wir, dessen Existenz angesichts der kurzen Aufmerksamkeitszyklen der Popwelt sowieso immer schon eine prinzipiell prekäre und ständig bedrohte ist. Das Stück vergeht nicht nur nach jeder Darbietung, sondern auch im uferlosen Ozean populärer Musik, vom künstlerisch-musikalischen Schaffen bleibt am Ende nichts übrig:

Same old song

Just a drop of water in an endless sea

All we do

Crumbles to the ground, though we refuse to see $^{6}$

Integrales Moment einer ,Pop-Haltung‘, so ließe sich sagen, ist das Wissen um diese Flüchtigkeit des eigenen Daseins - sowohl der Töne, die sich zwar auf Trägern sichern lassen, aber in der Regel irgendwann doch zu verhallen drohen, als auch der Pop-Subjekte selbst, der Stars, deren Hervorbringung immer ein auf eine bestimmte Dauer angelegtes, durch die Konjunkturzyklen der Popindustrie befristetes Unterfangen ist. Dieser Zusammenhang zwischen der Idee der transitoriness of life und der - diskursiv durchaus wirkmächtigen - Geste der transitoriness of pop ist für die Thematisierung von Vergänglichkeit im Pop nicht unbedingt konstitutiv. Er ist aber doch so wesentlich, dass er in verschiedenen Genres in ganz unterschiedlicher Form aufgerufen und - $\mathrm{u}$. a. durch die Entwicklung von diversen, wider des Vergehens angelegter Konservierungsstrategien populärer Musik - bearbeitet wird.

\section{Spurensuche: Variationen des Vanitas-Motivs in populärer Musik}

Ausgehend von den Beobachtungen von Wang und Kopf widmet sich diese Erkundung des Vanitas-Motivs im Pop zunächst der kulturellen Formation des Hip-Hop, denn gerade dort lässt sich die genannte Doppelbelegung des Motivs in der Tat nicht selten nachzeichnen. So etwa beim Gangsta-Rapper Sa4, dessen 2017 erschienener - und zusammen mit dem Rapperkollegen Gzuz aufgenommener - Song „Schall und Rauch“ das Motiv gleich in mehreren Hinsichten auf-

6 Kansas. „Dust in the Wind“. Point of Know Return. Kirshner / CBS und Epic Records, 1977. 
greift und dabei auch die eigene Musikerkarriere thematisiert. Die sich durch den Song ziehende catch phrase „Alles nur Schall, alles nur Rauch“, die bereits ein klassisches Element des Vanitas-Motivs aufruft, ${ }^{7}$ steckt dabei einerseits als rahmendes Motto das Thema der Lyrics ab und markiert andererseits gleich zu Beginn das Stück selbst als etwas Vorübergehendes. Dass in dessen Intro zunächst nur die erste Hälfte dieser catch phrase („Alles ist Schall“") eingespielt und mit einem starken Hall-Effekt unterlegt wird, der das Verschwinden des Klangs besonders hervorhebt, unterstreicht dieses selbstreflexive Moment.

Nachdem auf inhaltlicher Ebene zunächst vom „Leben mit Geld wie ein Rockstar“ mit „Auftritte[n], Partys, Termine[n]“ und „Mercedes“ geträumt wird ${ }^{8}$ - ein Traum, der von den kommerziell erfolgreichen Musikern Sa4 und Gzuz vermutlich sogar als realisiert gelten darf -, erfolgt im Refrain eine dem genretypischen Gestus durchaus angemessene Lamentatio der belanglosen, flüchtigen Erfahrungen auf dem Weg zum Erfolg: „Jede Frau, die Du einlädst | jeder Tag, den Du leidest | wie alles vorbeizieht | Alles nur Schall“9 ${ }^{\text {' }}$. Schließlich deutet die Rapper-Persona auf die Unausweichlichkeit der eigenen Vergänglichkeit hin, sowohl durch den Hinweis auf den durch Rauschzustände auf Hochtouren gebrachten Lebenswandel, der umso schneller zum Verschleiß führt („Die Zeit rennt, wir sind ausgebrannt | Jeden Tag im Rauschzustand | unantastbar wie Rauch vom Blunt“10), als auch durch die Thematisierung der stets virulenten Bedrohung der Existenz durch die in der ,Szene، ausgetragenen Straßenkämpfe:

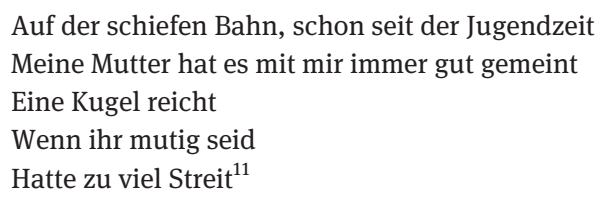

Die Antizipation eines solchen Endes durch einen Schusswechsel lässt sich dabei ebenfalls als Teil einer den Genrekonventionen angemessenen Selbstinszenierung verstehen. Denn auch die „Neun-Millimeter-Patronen“, die man für den Fall der Fälle bei sich trägt, sind nichts als „Dekoration | auf meinem Weg

7 Vgl. ausführlicher zum Motiv des Rauchs etwa Christian Wobbeler. „,Ein Rauch / diß Leben ist‘. Symbolgehalt und Selbstreferentialität von Rauch und Rauchen in zeitgenössischen Theaterinszenierungen“. Paragrana. Internationale Zeitschrift für Historische Anthropologie 27.2 [Themenheft ,Vanitas. Reflexionen über Vergänglichkeit in Literatur, bildender Kunst und theoretischen Diskursen der Gegenwart'. Hrsg. von Claudia Benthien und Victoria von Flemming] (2018): 249-267.

8 Sa4 (feat. Gzuz). „Schall und Rauch“. Neue Deutsche Quelle. 187 Straßenbande, 2017.

9 Ebd.

10 Ebd.

11 Ebd. 
zur Million“, ${ }^{12}$ wie der Song selber formuliert, „Alles nur Schall, alles nur Rauch“ eben.

Als Teil einer Authentizitätsbehauptung rahmt das schnelle Ableben also das ebenso schnelle Leben: Live Fast, Die Young. Der frühe Tod ist hier - ähnlich wie bei Idolen wie Elvis Presley, Marilyn Monroe, Tupac Shakur oder den im Alter von 27 Jahren verstorbenen ,Mitgliedern“ des sogenannten Club 27, darunter Janis Joplin, Jimi Hendrix, Kurt Cobain und Amy Winehouse - die Signatur eines bestimmten, immer schon riskanten, dadurch aber überaus faszinierenden Lifestyles, der gleichzeitig, ja paradoxerweise vielleicht sogar in besonderem Maße, das Überleben im popkulturellen Gedächtnis in Aussicht stellt. ${ }^{13}$ Denn letztendlich ist auch das Rapper-Dasein, so implizieren es die selbstreflexiven Momente des Songs, nichts weiter als das Resultat der Wiederholung von Gemeinplätzen mit vergleichsweise geringer Halbwertszeit.

Eine recht umfassende Beschäftigung mit dem Thema Tod und Vergänglichkeit findet sich auf dem Album Vanitas des Rappers Gaijin aus dem Jahr 2019. Schon die Namen einiger Stücke - etwa „Kommando Totenkopf (feat. Bagerly)“ oder „Rigor Mortis“, ${ }^{14}$ - weisen darauf hin, dass die Auseinandersetzung mit dem Ende hier zentral ist; hinzu kommt eine auf die gängigen visuellen Vergänglichkeitstopoi zurückgreifende Gestaltung des Albumcovers: Wir blicken in einen schemenhaft und in schwarz-weiß dargestellten, verwitterten, mindestens renovierungsbedürftig anmutenden leeren Raum, sehen einen leeren Stuhl mit defekter Lehne, von dem sich nur ahnen lässt, dass er unter dem Gewicht eines Menschen schlicht zusammenbrechen würde. Im oberen Drittel des Covers prangt zentral das Motiv des Totenkopfes mit angedeutetem Weizenkranz, ${ }^{15}$ auf der linken Seite gerahmt von rankenden Blüten, darunter die ebenfalls für das Vanitas-Motiv typische Darstellung von Schriftstücken (zwar nicht im Detail erkennbar, aber hier durchaus als Notenblätter deutbar) als Ausdruck des stets zum Scheitern verurteilten Versuchs der Fixierung von stimmlicher bzw. musikalischer Performance (Abb. 1).

Während Sa4 im Stück „Schall und Rauch“ sein Rapper-Dasein und dessen ephemere Momente thematisiert, steht hier im titelgebenden Stück „Vanitas“ nichts weniger zur Disposition als das Ende der bestehenden gesellschaftlichen

\footnotetext{
12 Ebd.

13 Vgl. Partridge 2015 (Anm. 4).

14 Gaijin. Vanitas. Freekmusick, 2019.

15 Zur Popularität und zum kommerziellen Erfolg des Totenkopf-Motivs vgl. das Kapitel „Death Sells: Vanitas en Vogue“ in Thomas Quast. Der Tod steht uns gut. Vanitas heute. Berlin: Nicolai, 2013. 43-67.
} 


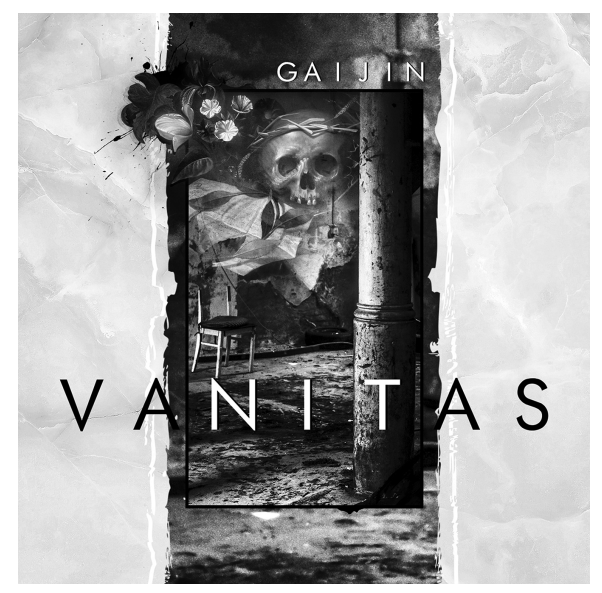

Abb. 1: Gaijin: Vanitas, Albumcover, 2019.

Ordnung, deren Korrumpiertheit sich in „babylonische[m] Treiben“ und „diabolische[m] Leiden“ äußert:

\author{
Euer Land steht für babylonisches Treiben \\ Und Eure schöne neue Welt für diabolisches Leiden \\ Mann, das sind komische Zeiten \\ Wenn man am Abgrund steht und Party macht \\ Aber wem sag ich das \\ Alles endet, Vanitas ${ }^{16}$
}

Der Song, den Gaijin zusammen mit dem Rapperkollegen Jot S aufgenommen hat, modelliert dieses Ende allerdings nicht als Bedrohung, sondern als Notwendigkeit zur Überwindung des Status Quo durch gewaltsamen Umsturz. Dazu inszeniert er den Dialog der beiden Rapper während der Durchführung eines Bombenattentats auf ein Bürogebäude. Beide sind - so lassen es uns die verzerrten Sprachaufnahmen zwischen den Strophen des Songs wissen - per Funkgerät miteinander verbunden und auf einer gemeinsamen Mission. Im „Sklavenwiderstand“, mit „der Fahne in der Hand“ soll das „Land [...] begraben“ werden, dessen Reichtum auf der Ausbeutung anderer beruht. ${ }^{17}$

Auch dieser Song, der uns durch die Inszenierung des Funkdialogs der beiden Attentäter an ihrer „Reise ohne Wiederkehr“18 teilhaben lässt, bedient sich

16 Gaijin (feat. Jot S). „Vanitas“. Vanitas. Freekmusick, 2019 (Anm. 14).

17 Ebd.

18 Ebd. 
also in der Beschreibung des Endes eines Rauch-Bildes: Anstatt von „Schall und Rauch“ ist hier von „Schutt und Asche“ die Rede, der Rauch ist Resultat von Waffengefechten und Bombenexplosionen. Der eigene Tod wird in diesem Szenario im Kampf für die gemeinsame Sache in Kauf genommen, um durch die beabsichtigte Abschaffung der existierenden Verhältnisse den als dringend nötig empfundenen Neuanfang zu ermöglichen: „für die verrückte Welt | Wird die Uhr wieder zurückgestellt“"19.

Dass das Ende des menschlichen Daseins - sowohl auf individueller als auch auf kollektiver Ebene - keine Bedrohung, sondern letztlich nur die schon lange absehbare und auch angemessene Konsequenz des eigenen Handelns oder des Handelns der anderen ist, wird auch im Genre des Punk immer wieder thematisiert. Die US-amerikanische Band Suicidal Tendencies etwa, deren Name programmatisch für die Ausrichtung ihrer Stücke ist, besingt regelmäßig die drastischen Konsequenzen psychosozialer Belastungen durch ökonomische Ausgrenzung. Auch Bands wie Bad Brains, Black Flag, Circle Jerks, The Dead Kennedys, D.O.A., Minor Threat, Misfits, Nine Inch Nails, Skinny Puppy oder State of Alert reagieren auf die dramatischen gesellschaftlichen Schieflagen in den USA bereits in den 1980er und 1990er Jahren mit dem Ausdruck von Hilf- und Hoffnungslosigkeit, die seelischen und körperlichen Verfall bedingen. ${ }^{20}$ Thematisiert wird der individuelle und gesellschaftliche Niedergang auch auf visueller Ebene - sei es auf Plattencovern, Stickern, Patches oder in Form von Körperschmuck, dessen ambivalente Wahrnehmung zwischen dem Hang zur Selbstzerstörung und der Kritik an etablierten Schönheitsidealen das provokative Potential des Punks verstärkt. Zentral ist dabei das Motiv des Totenkopfs als Teil des visuellen Szene-Repertoires, als Accessoire einer Subkultur sozusagen, das als „Indikator[] eines Vergangenen [...] wie einer fortwährenden Gegenwart (des im Prozess der Zersetzung Befindlichen) und des Zukünftigen (des finalen Zerfalls)“21 fungiert; als Symbol unaufhaltsamer Degeneration also, kombiniert mit einer ebenso zwingenden Perspektivlosigkeit unter dem Motto ,No Future‘. Mit dem Totenkopf wird allerdings nicht nur - oder nicht unbedingt - die Vanitas-Thematik aufgerufen.

19 Ebd.

20 Vgl. Partridge 2015 (Anm. 13), S. 4.

21 Katharina Sykora. „Enden und Verfliegen. Schädel, Insekten und zwei Temporalitäten der Vanitas in der zeitgenössischen Fotografie.“ Paragrana. Internationale Zeitschrift für Historische Anthropologie 27.2 [Themenheft ,Vanitas. Reflexionen über Vergänglichkeit in Literatur, bildender Kunst und theoretischen Diskursen der Gegenwart'. Hrsg. von Claudia Benthien und Victoria von Flemming] (2018): 191-207, S. 194. 
Im Kontext des Punk erfährt er eine Umdeutung, wird zur Markierung einer radikalen politischen Orientierung (sowohl links wie rechts). ${ }^{22}$

In anderen Fällen ist die Auseinandersetzung mit Vergänglichkeit und Vergeblichkeit in der Subkultur des Punk jedoch umfassender. Im 2012 erschienenen Song „State of Grace“ der US-amerikanischen Postpunk-Band Hot Water Music beispielsweise wird der eigene Status des Krankseins, des ,Sich-NichtMehr-Bewegen-Könnens‘ Ausgangspunkt für eine Gegenwartsdiagnose, in der auch die Gesellschaft als Ganze in den Blick gerät. Nicht nur man selbst fühlt sich „sick“, sondern irgendwie fühlt sich alles infiziert an, sodass es höchste Zeit für die richtige Medizin wäre - eine Medizin jedoch, die niemandem so richtig schmecken mag.

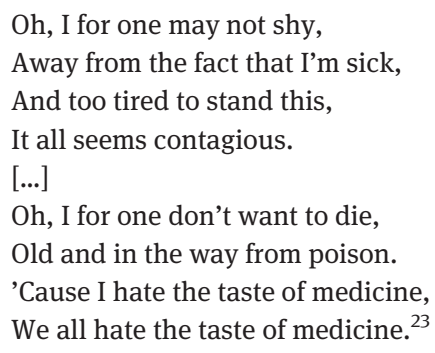

Zu der Angst vor dem Ende („I don’t want to die“) mischt sich auf der Ebene der Lyrics schließlich die Angst vor der Bedeutungs- und Nutzlosigkeit menschlicher Existenz: „I fear we're good for nothing“ - eine Existenz, der der „state of grace“, die Aussicht auf göttliche Gnade also, schon lange nicht mehr sicher ist, und die sich schlicht und ergreifend in Staub auflösen wird:

Our state of grace is crumbling,

I fear we're good for nothing,

And simply disintegrating into the terra firma. ${ }^{24}$

Im Videoclip zum Song blitzen Bilder junger und alter, teils auf medizinische Versorgung angewiesener, teils wie verkleidet anmutender Körper ebenso auf

22 Die nicht selten eher schemenhafte visuelle Gestaltung des Schädels bedient sich dabei üblicherweise der Ästhetik der angloamerikanischen Tätowiertradition - und umgekehrt. Auch in letzterer spielt der Totenkopf in unterschiedlichen Variationen eine zentrale Rolle. Als unter die menschliche Haut gebrachtes Motiv entfaltet er mit Blick auf seine materiell-physische Eigenschaft als Tätowierung seine Bedeutung als Symbol des Vergänglichen dabei auf eine besonders spannungsvolle Art und Weise. Vgl. dazu auch Quast 2013 (Anm. 15).

23 Hot Water Music. „State of Grace“. Exister. Rise Records, 2012.

24 Ebd. 
wie Close-Ups verfaulender, von Würmern zerfressener Früchte ${ }^{25}$ - eines im Rahmen der Vanitas-Thematik regelmäßig aufgerufenen Motivs. Der schnelle Schnitt analog zum Tempo des Stücks und der Wechsel zwischen Nahaufnahmen von Körpern und Obst unterstreichen die im Text des Songs thematisierte Prekarität menschlichen Daseins also auch auf visueller Ebene (Abb. 2 und 3).
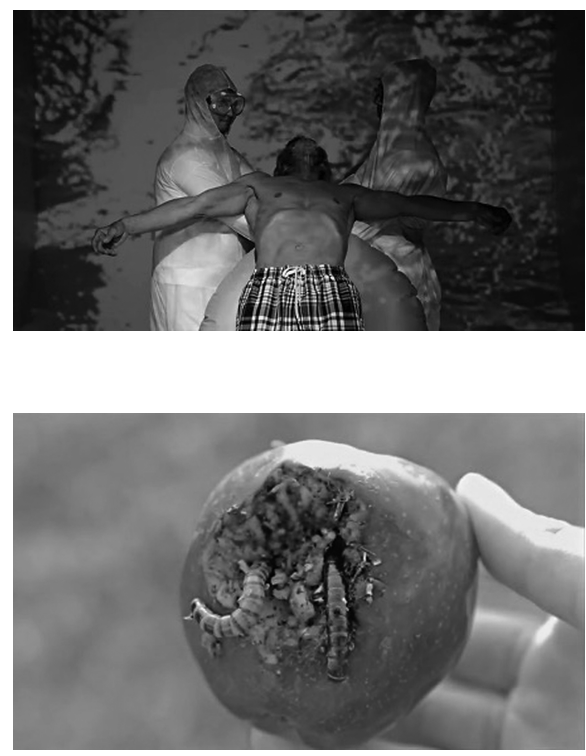

Abb. 2: Hot Water Music: State of Grace, Musikvideo, Regie: June Zandona, 2012.
Abb. 3: Hot Water Music: State of Grace, Musikvideo, Regie: June Zandona, 2012.

Die Angst vor dem Tod, die mit der Angst vor der Bedeutungslosigkeit des Daseins einhergeht, ist vielleicht auch deshalb Thema im Punk, weil Punk als Genre schon oft $\mathrm{zu}$ Grabe getragen wurde, und mindestens genauso so oft im Zuge von diesem und jenem Revival - wieder auferstanden ist. Die Band Crass stellt schon 1978 zum ersten Mal den Tod des Genres im Song „Punk is Dead“ auf ihrem Album The Feeding of the 5000 fest. Die in den Lyrics vorgetragene Autopsie ergibt Folgendes: Gestorben sei Punk am Ausverkauf einer einst als Gegenbewegung profilierten Musikkultur - in dem Moment also, in dem er zu „just another cheap product for the consumer's head“ geworden ist. Als „bubblegum rock on plastic transistors“ verliert Punk seine Existenzberechtigung:

Yes that's right, punk is dead,

It's just another cheap product for the consumer's head.

25 „Hot Water Music - State of Grace (Official Music Video)“ (2012). https://www.youtube. com/watch? $v=m U b X 80 X o 74 c$ (Zugriff am 23.10.2020). 


\begin{abstract}
Bubblegum rock on plastic transistors,
Schoolboy sedition backed by big time promoters.

CBS promote the Clash,

But it ain't for revolution, it's just for cash.

Punk became a fashion just like hippy used to be

And it ain't got a thing to do with you or me. ${ }^{26}$
\end{abstract}

Der Sündenfall, der letztlich das Ableben von Punk einleitet, ist hier schnell identifiziert: Die Kommerzialisierung der beiden damals schon ikonischen Vertreter der Subkultur - The Clash, namentlich genannt, und die Sex Pistols, hier aufgerufen durch eine Referenz auf den Gitarristen Steve Jones - ist der erste Schritt zur Abschaffung widerständigen Potentials. Der Moment, in dem es nur noch ums Geld geht (,it ain’t for revolution, it's just for cash“), ist der Moment einer Bankrotterklärung eines Genres, das belanglos wird, zu existieren aufhört, da seine für es konstitutive Position der Differenz durch die Warenhaftigkeit subkulturellen Widerstands erodiert. Die Vergeblichkeit der oppositionellen Haltung geht mit der Vergänglichkeit der Musikrichtung einher.

Davon, dass die Unumkehrbarkeit dieses Prozesses immer wieder zur Disposition gestellt wird, zeugen eine ganze Reihe von Revivals des Punk, eine Form des ,Wieder-Holens', die sich gegen Endlichkeit und Endgültigkeit richtet. Drei Jahre nach „Punk is Dead“ etwa stellt die Band The Exploited die Wiederauferstehung des Genres fest: Punk's Not Dead heißt deren erstes Album, das im Jahre 1981 erscheint und als Reaktion auf den Song von Crass lesbar ist. ${ }^{27}$ Auch im Punk also lässt sich die auf sich selbst bezogene Variante des VanitasMotivs beobachten. Allerdings steht in dieser kulturellen Formation nicht so sehr die Flüchtigkeit medialer Aufmerksamkeit oder des Ruhms erfolgreicher Platten im Mittelpunkt, sondern vielmehr die ständige Gefahr der Absorption subkulturellen Widerstands durch den sogenannten Mainstream - der Tod geht hier von der im Moment der Vereinnahmung erzeugten Erfahrung politischer Belanglosigkeit aus.

Auch für das Punk-Revival der 1990er Jahre, genauso wie für die vielen Ausläufer des sogenannten Post-Punk der 2000er Jahre, ist die Reflexion der existentiellen Bedrohung der Belanglosigkeit zentral. Die ,Einsicht‘ in die eigene Vergeblichkeit sowie Vergänglichkeit und die ,Absicht' der eigentlich immer schon zum Scheitern verurteilten Versuche ihrer Überwindung sind dabei zwei sich gegenseitig bedingende, aufeinander bezogene Momente. Sie sind konstitu-

26 Crass. „Punk is Dead“. The Feeding of the 5000. Crass Records, 1978.

27 Vgl. Martin Butler. „Punk is Dead. Or is it? On Strategies of Subcultural Positioning in / and the (Re-)Making of the Punk Movement“. Sonic Politics: Music and Social Movements in the Americas. Hrsg. von Olaf Kaltmeier und Wilfried Raussert. New York, NY: Routledge, 2019. 205-215. 
tiv für den Prozess der Auseinandersetzung mit einer stets prekären, zeitlich befristeten, durch Verschiebungen in Aufmerksamkeitsökonomien hergestellten, aber eben auch genau dort wieder aufs Spiel gesetzten Existenz. ${ }^{28}$ Die retrospektive, zuweilen romantisch verklärte Betrachtung der eigenen musikalischen Biografie ist sicher eine der Strategien, diese beiden Momente zumindest irgendwie ,in Schach zu halten'.

Das im Jahre 2012 veröffentlichte Album Days Go By der kalifornischen Punkband The Offspring beispielsweise thematisiert das musikalische Altern nicht nur im Titel, sondern auch auf dem Albumcover, das durch die Abbildung zweier Generationen, zwischen denen mindestens eine dritte liegt, die Unausweichlichkeit des Vergehens illustriert: ${ }^{29}$ Man sieht einen Jungen und einen älteren Mann auf einer Bank sitzen, die Vermutung, dass es sich bei letzterem um den Großvater des ersteren handelt, liegt nahe. Die beiden sind an jeweils einen Rand der Bank gedrängt, wirken abgewandt, der ältere Mann schaut in die Kamera, während die Blicke des Jungen zu schweifen scheinen. Die Farbgebung ist in dunklen Erd- und Schwarztönen gehalten, eine in Nebel getauchte, nasskalt wirkende spätherbstliche Waldkulisse als Hintergrund untermalt die visuelle Thematisierung von Vergänglichkeit (Abb. 4). Auch der Titeltrack des Albums, „Days Go By“, fokussiert das Älterwerden und zieht Bilanz. Hier heißt es:

\author{
All our yesterdays are pictures lost in time \\ Never enough \\ Towers crumble to dust \\ Looking back on the moments of our lives \\ All your anger, all your hurt \\ Doesn't matter in the end \\ Those days go by \\ And we all start again \\ What you had and what you lost \\ They're all memories in the wind \\ Those days go by \\ And we all start again ${ }^{30}$
}

28 Vgl. Martin Butler und Arvi Sepp. „Punk’s not Dead. Erinnerungen als Strategie der Abgrenzung und Neuorientierung einer (totgeglaubten) Subkultur“. Siegener Periodicum zur internationalen empirischen Literaturwissenschaft 24 (2008): 285-296.

29 Das Cover erinnert in der gemeinsamen Abbildung der Generationen an die frühneuzeitliche Darstellung der drei Lebensalter etwa auf Hans Baldung Griens Gemälde Die drei Lebensalter und der Tod (1541-1544).

30 The Offspring. „Days Go By”. Days Go By. Columbia Records (Sony Music), 2012. 


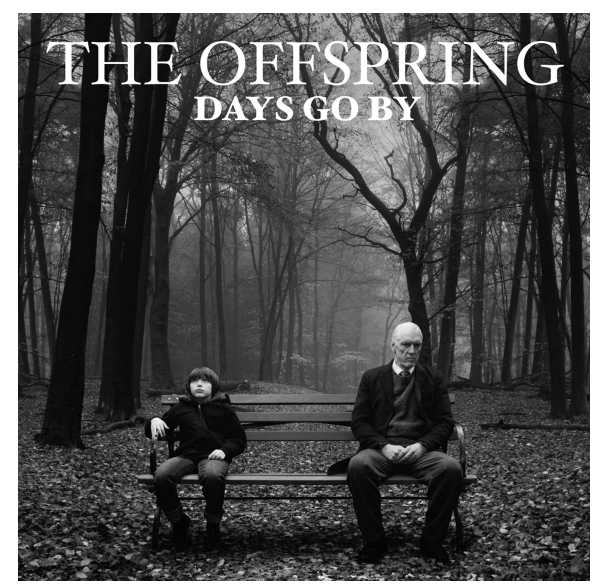

Abb. 4: The Offspring: Days Go By, Albumcover, 2012.

Wut und Schmerz - „all your anger, all your hurt“ - zählen doch am Ende nichts. All das, was war, ist Windhauch - „memories in the wind“. Dennoch oder gerade deswegen - fängt man aber, so die Lyrics, doch immer wieder von vorne an, „And we all start again“. Das ,Wieder-Holen“, das ,Wieder-von-VornAnfangen', ist folglich auch hier als gegenläufiges, das Vergangene, oder das Vergehende, aufhebendes Moment zumindest angedeutet.

So wie der Titeltrack allein lässt sich auch das gesamte Album als ein Versuch der wiederholten und wiederholenden Markierung überzeitlicher Bedeutung des eigenen Tuns verstehen: Schließlich sichert man sein Werk - und damit auch sich selbst - auf einem Tonträger, arbeitet an der Archivierung im musikalischen Gedächtnis einer Subkultur. Sieben Jahre nach Erscheinen des Best-Of-Albums der Band, das sich bereits als Versuch gegen das Vergessen lesen lässt, changiert also auch Days Go By zwischen der Erkenntnis des unentrinnbaren Endes aller Dinge und der Motivation, dieses Ende, zumindest das der eigenen musikalischen Karrieren, doch noch - und mal wieder - ein Stückchen weiter weg zu schieben, aufzuhalten, herauszuzögern.

Auch die Flucht in die Gegenwart, ins Hier und Jetzt, ist als Strategie und Thema im Pop anzutreffen, um mit Vergänglichkeit und Vergeblichkeit umzugehen. Als Reaktion auf die Einsicht in das nahende und unaufhaltsame Ende und als Gegenbewegung zu einer doch immer deutlicher ausgeprägten Vergangenheitsbesessenheit des Pop wird daher der Gestus des carpe diem ins Popvokabular übersetzt. „Alles ist Jetzt“, singt etwa Axel Bosse auf seinem gleichnamigen Album aus dem Jahre 2018: 
Und alles ist jetzt
Es ist alles, alles jetzt
Das Leben ist kurz
Zu kurz für ein langes Gesicht
Und Stück für Stück kommt das Lachen zurück
Und die Freude und der Hüftschwung und das Glück ${ }^{31}$

Vielleicht, so ließe sich argumentieren, bleibt dem Pop ja auch nichts anderes übrig als das Hier und Jetzt - und zwar nicht nur aufgrund der kurzen Dauer der bereits angesprochenen Aufmerksamkeitszyklen im Popkulturbetrieb, sondern auch aufgrund der Tatsache, dass sein zentrales Medium, der Klang nämlich, per se als Verschwindendes, Verhallendes, verstanden werden muss. Auch diese Dimension populärer Musik, die erst in der Performance akustisch Gestalt annimmt, trägt sicher zu ihrer (Selbst-)Wahrnehmung als flüchtig bei. Aufnahmeund Abspieltechnologien wie Reverb oder Fading Out thematisieren diese Flüchtigkeit und versuchen sie gleichzeitig abzustellen: durch die Konservierung auf Tonträgern oder durch die Repeat- und Shuffle-Funktionen in Streaming-Portalen. Während die einzelnen Stücke auf einer Playlist - notwendigerweise und unaufhaltsam - vorübergehen, sorgen Algorithmen für das Gefühl der Endlosigkeit des Musikstroms.

,Vorübergehen' und ,Auf-Dauer-Stellen` sind auch die beiden Momente, die in Johnny Cashs retrospektiver Auseinandersetzung mit dem eigenen Schaffen angesichts der Einsicht in das bevorstehende Ende zentral gestellt sind. In seiner Coverversion des Nine-Inch-Nails-Songs „Hurt“ aus dem Jahre 2002 nämlich lässt der gealterte Countrysänger seine Karriere Revue passieren, sowohl auf Ebene der Lyrics als auch im dazugehörigen Videoclip, der Motive des Vergänglichen aufgreift und dabei gleichzeitig als Stillstellung fungiert, die als Form der audiovisuellen Musealisierung dem Vergessenwerden entgegenzuwirken versucht. ${ }^{32}$ Der Rückgriff auf a-Moll in der musikalischen Inszenierung dieses Endes ist dabei laut Cristina Pileggi (im Anschluss an Alfred Stengers Einordnungen von Tonarten) von besonderer Bedeutung: denn „[w]ird das Musikvideo als Cashs Abschiedswerk rezipiert, so grenzt hier die Verwendung der

31 Axel Bosse. „Alles ist Jetzt“. Alles ist Jetzt. Vertigo Berlin, 2018. Zur Jetzt-Bezüglichkeit als zentrales Merkmal des Pop vgl. Eckart Schumacher. Gerade Eben Jetzt. Schreibweisen der Gegenwart. Frankfurt a. M.: Suhrkamp, 2003.

32 „Johnny Cash - Hurt (Official Music Video)“ (2019). https://www.youtube.com/watch?v= 8AHCfZTRGiI (Zugriff am 23.10.2020). Für eine umfassende, intermedial angelegte Analyse der (auto)biographischen Inszenierungsstrategien des Clips vgl. Cristina Pileggi. „,My Empire of Dirt‘. Die audiovisuelle Biographie in Johnny Cashs Musikclip Hurt“. Lied und Populäre Kultur / Song and Popular Culture 62 (2017): 241-258. 
Tonart a-Moll schon fast an Jean Sibelius’ ,Todessymbol““33. Besungen wird der körperliche Zerfall genauso wie der Zerfall eines Imperiums, eines „empire of dirt“, also einer kommerziell durchaus erfolgreichen, aber doch - so impliziert es zumindest der Begriff dirt - persönlich nicht immer gradlinigen Musikerkarriere, deren Redlichkeit sich in Frage stellen ließe. Selbstzweifel (,what have I become?“34) paaren sich mit dem Schmerz, der den Tod ankündigt, und gleichzeitig ist der Schmerz das letzte und deutlichste Lebenszeichen: „I hurt myself today | to see if I still feel | I focus on the pain | the only thing that's real“35. Im Videoclip reihen sich Symbole der Vanitas aneinander: verlassene Räume, zerbrochene Bilderrahmen, ein Teller mit verderbendem Obst, Würmer, dazwischen Filmmaterial, das auch einer Dokumentation über Cashs Leben und Werk entnommen sein könnte. ${ }^{36}$ Cashs Stimme als Sänger und seine Stimme in der US-amerikanischen Countryszene (und darüber hinaus), so lässt es uns der Clip hören und sehen, wird brüchig und verschwindet - und sie bleibt, oder soll bleiben, nicht zuletzt manifestiert durch den Clip selbst, als Teil einer Hinterlassenschaft und als Erinnerungsort.

\section{Schlussakkord}

Zum Schluss nun steht der Versuch, diese Beobachtungen in größere Zusammenhänge einzuordnen und zugleich auch Impulse für eine vertiefte Diskussion über Pop und Vergänglichkeit zu liefern: Die immer größere Anzahl solcher Erprobungen des Entrinnens, der Flucht vor der Vergänglichkeit in vollem Bewusstsein über die Endlichkeit des eigenen Schaffens lassen sich wohl auch auf die im öffentlichen und akademischen Diskurs mittlerweile etablierte, wenn auch nicht unstrittige Argumentationsfigur des Endes der Popkultur beziehen. Das, was Jeremy Beadle schon in den 1990er Jahren mit der Frage „Will Pop Eat Itself?“37 in seinem gleichnamigen Buch ankündigte, eine Entwicklung also, in der Popkultur - als ewig wiederkäuende Kopiermaschine - sich sozusagen selbst verdaut und sich damit ein Ende bereitet, ist seit einigen Jahren als Tendenz zur ,Retromanie ${ }^{38}$ lanciert worden: Eine im Spätkapitalismus unvermeidbare, ja

33 Pileggi 2017 (Anm. 32), S. 255.

34 Johnny Cash. „Hurt“. The Man Comes Around. Mercury (Universal Music), 2002.

35 Ebd.

36 Vgl. ebenso Pileggi 2017 (Anm. 32), S. 247.

37 Jeremy Beadle. Will Pop Eat Itself? Pop Music in the Soundbite Era. London: Faber \& Faber, 1993.

38 Vgl. Reynolds 2012 (Anm. 5). 
erst durch dessen globalisierte und sich stets beschleunigende Warenströme bedingte Form der ständigen Selbstreferenz, die zu Ideen- und Innovationslosigkeit führt bzw. darauf beruht, und damit, so zumindest die Vertreter^innen dieser These, ein für Pop existentielles Problem wird. Pop besteht, so Reynolds, nur noch in und aus der endlosen, in mehr oder weniger großen Schleifen der Selbstbezugnahme drehenden Retrospektive: Revivals, Best-ofs, Reunions, Remakes, Coverversionen, Mash-Ups, Shreds, Spoofs - all diese Formen kulturellen Ausdrucks sind Wiederholungsformen. „In keiner Epoche der Popgeschichtsschreibung war die Rückbesinnung auf schon mal Dagewesenes so gegenwärtig wie in den vergangenen zehn Jahren“39, kommentiert Rabea Weihser in ihrer Rezension zu Reynolds Retromania. „Vor Lana del Rey waren Kitty, Daisy \& Lewis mit ihrem originalgetreuen Rock'n'Roll, Hurts mit ihrem kühlen 80er-JahreSynthiepop, Adele mit ihrem 60er-Jahre-Soul, The XX mit ihrem Post-Punk-Appeal. Die Reihe ließe sich endlos fortsetzen “40. Und Reynolds selbst verwendet in der Beschreibung dieser Wiederkehr des immer Gleichen eine Sprache, die die Entwicklung der Popmusik mit körperlichem Verfall in Verbindung bringt. Das „schleichende Verschwinden“ der Gegenwartsbezogenheit des Pop im neuen Jahrtausend, in dem „der Pulsschlag des JETZT sich mit jedem Jahr schwächer anfühlte“, würde sich daran bemerkbar machen, dass „man unter der straffen Haut und den rosa Wangen junger Bands das schlaffe graue Fleisch alter Ideen durchschimmern sah". ${ }^{41}$ Es dauert also nicht mehr lange bis zum Exitus - so impliziert es zumindest Reynolds’ Beobachtung eines fortschreitenden Alterungsprozesses.

Nun könnte man sicher fragen, ob das Wiederholende, das Serielle, das Iterative, nicht immer schon Produktionsprinzip des Pop war und der innovative Charakter eine bloße Unterstellung von Originalitätsromantiker^innen. Gewinnbringender als dieser Frage nachzugehen erscheint es jedoch, den Diskurs zum Ende des Pop mit einer Bewegung im Pop selbst zu korrelieren, die wohl seit der Jahrtausendwende ein besonderes Momentum zu entfalten scheint. Dieser Streifzug, der - geleitet von der Beobachtung der selbstreflexiven Dimension des Pop - Variationen der Thematisierung von Vergänglichkeit in unterschiedlichen intermedialen Konstellationen des Pop nachgegangen ist, hat diese Korrelation aufzuzeigen versucht. Erkenntnisleitend war also nicht so sehr die Frage nach den spezifischen gesellschaftlichen Umständen, die die Fokussierung auf das Vergängliche bedingen - die von Wang und Kopf zu Anfang zitierte Diag-

39 Rabea Weihser. „Pop am Rande der Erschöpfung“. ZEIT online (14.10.2011). https://www. zeit.de/kultur/musik/2011-10/retromania-simon-reynolds (Zugriff am 23.10.2020).

40 Ebd.

41 Reynolds 2012 (Anm. 5), S. 17-19. 
nose einer Enttabuisierung des Themas Tod als Grund für die gesteigerte Aufmerksamkeit wäre daher weiter zu diskutieren und zu differenzieren. Vielmehr zielte diese Erkundung darauf ab, die Bedingungen der Thematisierung von Vergänglichkeit auch, vielleicht sogar vor allem in der für Popmusik konstitutiven Transitorität, also seiner vielfach diskursiv attestierten, aber durchaus auch empirisch beobachtbaren Schnell- oder Kurzlebigkeit $\mathrm{zu}$ suchen, die in Form metareferentieller Bezugnahmen auf sich selbst immer wieder - und dabei mehr oder weniger explizit - zum eigentlichen Gegenstand des Nachdenkens über das Ende wird.

„All we are is Dust in the Wind“ - „Das ist alles Windhauch“ (Koh. 1,2 $\left.{ }^{\mathrm{EU}}\right){ }^{42}$ Sicher ist dieses selbstreflexive Moment nicht überall gleich ausgeprägt bzw. ausgelegt. Und sicher ist auch nicht überall dort ein umfassender Rekurs auf das Vanitas-Motiv vorzufinden, wo er dem ersten Anschein nach vorliegt. Zumindest dann nicht, wenn man von einem gesichert beschreibbaren Kern eines barocken Motivs ausgehen möchte, dessen Konturen dann verfransen, wenn nur auf der ,Oberfläche' darauf Bezug genommen wird. ${ }^{43}$ Letzlich findet sich das lose Spiel der Referenzen und Selbstreferenzen als Konstruktionsprinzip des Pop - bezogen auf die Themen Vergänglichkeit und Tod - auch in etlichen anderen Musikstilen und -kulturen, über deren Aneignungen und Umcodierungen des Motivs man sicher auch noch differenzierter sprechen könnte; man denke etwa an ,übliche Verdächtige“ wie Death Metal, Death Doom, Gothic Metal oder Dark Wave, deren Präferenz für Todesmotive auf textlicher, visueller und performativer Ebene eine Beschäftigung mit Vanitas ja schon ,qua Genre' naheliegt.

Weiter nachzudenken wäre sicher auch über die Frage, ob das Sinnieren über Vergänglichkeit (der Musik, des Ruhms, des Lebens) ein spezifisch männlicher Gestus in der Popmusik ist. Die hier ausgewählten Beispiele, in und mit denen das Vanitas-Motiv im Repertoire des Pop aktualisiert wird, legen dies zumindest nahe. Zugegeben: Schon die besprochenen Genres (HipHop, Punkrock und Country) sind im weitgehend heteronormativen Diskursfeld des Pop ohnehin

$42 \mathrm{Zu}$ den Implikationen der Wind-Metaphorik bei Kohelet sowie deren Variationen in verschiedenen Übersetzungen dieser Passage vgl. Dorothea Scholl. „,Vanitas vanitatum et omnia vanitas'. Das Buch Kohelet in der europäischen Renaissance- und Barocklyrik und Emblematik“. Bibeldichtung. Hrsg. von Volker Kapp und Dorothea Scholl. Berlin: Duncker \& Humblot, 2006. 221-260, S. 224-225. Ihre Verweise auf Übersetzungen als „Rauchfahne“ oder „Rauchwolke“ (ebd. S. 224) vermögen sicher auch die Lektüre des oben besprochenen Sa4-Songs „Schall und Rauch“ produktiv anzureichern.

43 Vgl. dazu die Ausführungen zu „Entleerung und Resemantisierung“ von Claudia Benthien und Victoria von Flemming. „Einleitung“. Paragrana. Internationale Zeitschrift für Historische Anthropologie 27.2 [Themenheft ,Vanitas. Reflexionen über Vergänglichkeit in Literatur, bildender Kunst und theoretischen Diskursen der Gegenwart‘. Hrsg. von dens.] (2018): 11-35, S. 24-30. 
stark männlich konnotiert; folglich ließe sich gerade das leidend-leidenschaftliche Besingen des eigenen Vergehens als Element der Konzeption einer - eben männlichen - Heldenfigur verstehen, deren spezifische Art und Weise des Umgangs mit dem nahenden Ende - je nach Genre - sowohl an hegemoniale Männlichkeitsideale heroischer Furchtlosigkeit (etwa im Gangsta-Rap) anknüpft als auch an der Vorstellung eines homme fragile (etwa im Postpunk) ausgerichtet ist.

Mit einer solchen Perspektive, die erstens die Spezifik des Selbstbezugs populärer Musik als Konstruktionsprinzip berücksichtigt, zweitens die Genrekonventionen als Parameter in den Modellierungen des Vanitas-Motivs in Rechnung stellt und drittens nicht zuletzt die mit unterschiedlichen Konstellationen des Pop einhergehenden Normativitäten in den Blick nimmt, lassen sich die Formen und Funktionen der Thematisierung von Vergänglichkeit und Vergeblichkeit in populärer Musik gerade auch vor dem Hintergrund der angesprochenen Debatten um ihre scheinbar wachsende Vergangenheitsbesessenheit systematischer beleuchten.

\section{Abbildungsnachweise}

Abb. 1 Freekmusick, 2019. Fotograf: Frank Bollen. Grafik: Benjamin Kraus

Abb. 2-3 Rise Records, 2012. https://www.youtube.com/watch?v=mUbX80Xo74c (Zugriff am 01.02.2021)

Abb. 4 Columbia Records, 2012. Fotograf und Artwork: deadskinboy 